\title{
Validity of the Body Mass Index and Fat Mass Index as an Indicator of Obesity in Children Aged 3-5 Year
}

\author{
Chieko Eto $^{1)}$, Shuichi Komiya ${ }^{2)}$, Takehira $\mathrm{Nakao}^{2)}$ and Kazutoshi Kikkawa ${ }^{3)}$ \\ 1) Faculty of Health and Social Welfare, Nishikyushu University \\ 2) Graduate School of Human-Environment, Kyushu University \\ 3) Department of Health and Physical Education, Hiroshima Prefecture University
}

\begin{abstract}
The validity of the BMI and fat mass index (FMI) as indicators of obesity was evaluated in a group of $3-5 \mathrm{yr}$ old $(n=486)$ children. Bioelectrical impedance analysis (BIA) was measured (using $50 \mathrm{kHz}$ and tetrapolar electrodes) in order to calculate percent fat mass $(\% \mathrm{FM})$ and FMI (fat mass/stature squared). For boys, obesity was defined as $\geqq 20 \% \mathrm{FM}$. For girls, the cutoff for obesity was $\geqq 25 \% \mathrm{FM}$. However, obesity was defined as a BMI at or above the 90th percentile of age- and sex-specific data in this study. The percentile cutoffs for FMI were the same as for BMI using the same sample. There were correlations between BMI or FMI and \%FM, but there was no significant correlation between BMI or FMI and stature. Therefore, it appears that both the BMI and FMI in this study are far more useful indices with which to assess obesity, and are reasonable indicators of fatness. However, with the use of $\%$ FM by BIA as the criterion for obesity, BMI and FMI had high specificities $(95.5-96.4 \%$ for BMI and $99.5-100 \%$ for FMI) and lower but variable sensitivities (30.4-37.5\% for BMI and $42.9-68.8 \%$ for FMI). Thus, almost all children who were not obese were classified correctly. In contrast, many obese children were not correctly identified by BMI and FMI. Therefore, we conclude that BMI should be used with caution as an indicator of childhood obesity. The new recommendations based on the FMI approach for defining childhood obesity are associated with a level of sensitivity that is somewhat higher than that of the BMI approach. Caution should, however, be used in generalizing from the findings in this study, and a further investigation of the issue is required. $J$ Physiol Anthropol Appl Human Sci 23 (1): 25-30, 2004 http://www.jstage.jst.go.jp/en/
\end{abstract}

Keywords: body mass index, fat mass index, obesity, sensitivity, specificity, young children

\section{Introduction}

Obesity is defined as an excess of body fat mass (FM).
However, FM is extremely difficult to measure in young children, because accurate techniques require a high degree of the subject compliance. Therefore, the majority of large-scale studies of increasing FM in children used a body mass index (BMI, $\mathrm{kg} / \mathrm{m}^{2}$ ) as an index of body fatness (Troiano et al., 1995; Reilly and Dorosty, 1999), and BMI has been recommended as the best measurement for defining obesity in the child population (Dietz and Robinson, 1988). Currently, BMI is used widely because of the relative ease and accuracy of the basic measurements (Himes and Dietz, 1994).

Theoretically, BMI represents an index of body mass independent of stature such that at any age, greater relative body mass may be attributed to the increased body fatness. However, the relationship between BMI and fatness in childhood has received insufficient attentions. The major shortcoming of the BMI is that the actual composition of body mass is not taken into account. The body mass comprises both the fat-free mass (FFM) and FM, and both of these components can vary among individuals. Wells (2000) demonstrated previously that a between-subject variability in FFM is an important source of a variability in BMI in children. Although FFM remains relatively stable throughout adult life in healthy people, there are age and gender differences (Forbes and Reina, 1970), and FFM varies directly with stature. The body mass is reasonably correlated with FM but is also highly correlated with stature, which is weakly correlated with FM.

Therefore, body mass adjusted for stature is a far more useful index with which to assess obesity and is a reasonable indicator of fatness. However, the adjustment for stature does not completely eliminate the stature effect. For the first National Health and Nutritional Examination Survey (NHANES I), the correlation between the stature and the BMI was approximately 0.30 for children (Garn et al., 1986). Before $1 \mathrm{yr}$ and after $6 \mathrm{yr}$, both body mass and stature increase with growth. Therefore, the coefficients of correlation between BMI and stature are weak and sometimes negative from 1 to $6 \mathrm{yr}$, and are higher and positive before $1 \mathrm{yr}$ and after $6 \mathrm{yr}$ (RollandCachera et al., 1982). Thus, factors such as growth make 
definitions of obesity more complex in children.

The fat mass percent $(\% \mathrm{FM})$ is influenced by the relative amount of FFM, and, like BMI, is not an independent index of body fatness. The importance of this issue is demonstrated by the fact that, even in the general population, variability between subjects in relative FFM size is two-thirds of the variability in fatness (Wells, 2000). Obesity results in additional FFM as well as additional FM (Griffiths et al., 1990), and the expression of body fatness in obese children as $\%$ FM will underestimate the absolute amount of FM gained, and conceal the variability in FFM. To resolve this issue, both FFM and FM can be normalized for stature. Since BMI is the established index of body mass (BM) relative to stature (ST):

$$
\mathrm{BMI}=\mathrm{BM} / \mathrm{ST}^{2}=\mathrm{FFM} / \mathrm{ST}^{2}+\mathrm{FM} / \mathrm{ST}^{2} .
$$

These two indices, known as the fat-free mass index (FFMI, $\mathrm{kg} / \mathrm{m}^{2}$ ) and fat mass index $\left(\mathrm{FMI}, \mathrm{kg} / \mathrm{m}^{2}\right)$, are both discrete and adjusted for body size (Van Itallie et al., 1990). Just as BMI is useful in evaluating the body mass of individuals of different statures, so is the FMI potentially useful in helping clinicians interpret FM data from obese individuals who differ in stature.

Furthermore, the BMI has limitations; it tends to have high specificity as well as variable sensitivity in children (Himes and Bouchard, 1989; Marshall et al, 1991). The purpose of the present study is to evaluate the sensitivity and specificity of the BMI and FMI as indicators of obesity in groups of children ranging from $3-5 \mathrm{yr}$.

\section{Methods}

The subjects included 233 boys and 253 girls aged 3-5yr. The subjects' physical characteristics are summarized in Table 1. The subjects were a sample of recruited from the metropolitan area of Fukuoka, Japan. The subjects were free of serious diseases and took no drug or medication. Children were recruited by word of mouth through kindergarten teachers. The nature and purpose of the investigations were fully explained to each subject's parents before the study. The parents provided informed consent before the testing began.

The body mass was measured on the subject in light clothing and without shoes to the minimum of $0.02 \mathrm{~kg}$ on a balance beam metric scale, and was followed by measurement of stature to the minimum of $0.1 \mathrm{~cm}$ via a fixed, wall-mounted metric ruler. BMI (body mass, $\mathrm{kg} / \mathrm{stature}, \mathrm{m}^{2}$ ) was calculated for all subjects. The skin-fold thickness was determined to the minimum of $0.2 \mathrm{~mm}$ using a Harpenden skin-fold caliper. Measurements were taken at the right triceps and subscapular.

The body composition was measured by bioelectrical impedance analysis (BIA). All BIA measurements were performed in $2-3 \mathrm{~h}$ after the last meal. The whole-body resistance $(\mathrm{R})$ was measured with four surface electrodes placed on the right wrist and ankle, as previously described (Lukaski, 1986). Briefly, the principle was based on the application of an electrical current of $50 \mathrm{kHz}$ and $0.5 \mathrm{~mA}$ produced by a generator (TP-95K, Toyo Physical, Fukuoka, Japan) and applied to the skin using adhesive electrodes (Red Dot-2330, 3M Health Care, USA) with the subject lying supine (Houtkooper, 1996). The skin was cleaned with $76.9-81.4 \mathrm{vol} \%$ alcohol. Before each testing session, the calibration of the unit was checked using a $400-\Omega$ precision resistor. The FFM equation used here for children is based on

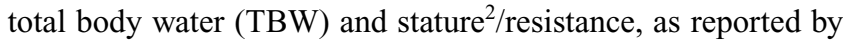
Goran et al. (1993). FM is then calculated as the difference between the body mass and FFM.

Both FFM and FM were divided by stature $^{2}$ to FFMI and FMI, respectively, as described previously (Van Itallie, et al., 1990).

The validity of using BMI or FMI to diagnose obesity was determined. The sensitivity and specificity were calculated using the equations of Himes and Bouchard (1989). Cutoffs at specific levels of \%FM were used to determine obesity (Owen, 1988 ). For boys, obesity was defined as $\geqq 20 \%$ FM. For girls, the cutoff for obesity was described as $\geqq 25 \% \mathrm{FM}$. However, BMI are also often used in many countries although the cutoff points vary between 85 and 97 percentile (Guillaume, 1999). In this study, obesity was defined as a BMI at or above the 90th percentile of age- and sex-specific data. The percentile cutoffs

Table 1 Age, anthropometry and body composition of the subjects

\begin{tabular}{|c|c|c|c|c|c|}
\hline & Boys $(n=233)$ & (range) & Girls $(n=253)$ & (range) & $p^{1)}$ \\
\hline Age, yr & $4.3 \pm 0.7$ & $(3.0-5.0)$ & $4.4 \pm 0.7$ & $(3.0-5.0)$ & NS \\
\hline Stature, $\mathrm{cm}$ & $104.9 \pm 6.3$ & $(88.2-120.6)$ & $105.3 \pm 6.1$ & $(84.5-122.2)$ & NS \\
\hline Body mass, kg & $17.29 \pm 2.80$ & $(11.14-30.05)$ & $17.01 \pm 2.20$ & $(11.98-23.68)$ & NS \\
\hline \multicolumn{6}{|l|}{ Skinfold thickness, mm } \\
\hline Triceps & $11.0 \pm 2.6$ & $(4.8-21.0)$ & $11.7 \pm 2.3$ & $(6.6-19.8)$ & $* *$ \\
\hline Subscapular & $6.7 \pm 2.2$ & $(3.6-19.0)$ & $7.3 \pm 2.4$ & $(4.0-21.4)$ & $* *$ \\
\hline Fat-free mass, $\mathrm{kg}$ & $14.00 \pm 2.19$ & $(8.19-22.13)$ & $13.36 \pm 1.79$ & $(8.50-18.05)$ & $* * *$ \\
\hline Fat mass, $\mathrm{kg}$ & $3.29 \pm 1.21$ & $(1.46-10.19)$ & $3.65 \pm 1.13$ & $(1.72-8.47)$ & $* * *$ \\
\hline Body mass index, $\mathrm{kg} / \mathrm{m}^{2}$ & $15.7 \pm 1.4$ & $(13.0-21.8)$ & $15.3 \pm 1.2$ & $(13.0-20.1)$ & * \\
\hline Percent fat mass, $\%$ & $19.0 \pm 5.2$ & $(10.0-36.0)$ & $21.5 \pm 5.4$ & $(12.1-38.0)$ & $* * *$ \\
\hline Fat-free mass index, $\mathrm{kg} / \mathrm{m}^{2}$ & $12.7 \pm 1.0$ & $(10.0-15.3)$ & $12.0 \pm 0.9$ & $(9.1-15.8)$ & $* * *$ \\
\hline Fat mass index, $\mathrm{kg} / \mathrm{m}^{2}$ & $3.0 \pm 1.0$ & $(1.40-7.90)$ & $3.3 \pm 1.0$ & $(1.6-7.2)$ & $* * *$ \\
\hline
\end{tabular}

\footnotetext{
${ }^{1)}$ Gender difference * $p<0.05 ; * * p<0.01 ; * * * p<0.001$
} 
Table 2 Intercorrelation of stature, body mass, triceps, subscapular, fat-free mass, fat mass,body mass index, percent fat mass, fat-free mass index, and fat mass index

\begin{tabular}{lrrrrrrrrrr}
\hline & ST & BM & Tric & Subs & FFM & FM & BMI & \%FM & FFMI & FMI \\
\hline ST & & 0.816 & 0.089 & 0.289 & 0.862 & 0.334 & 0.123 & -0.090 & 0.186 & -0.027 \\
BM & 0.789 & & 0.447 & 0.652 & 0.912 & 0.671 & 0.667 & 0.212 & 0.522 & 0.385 \\
Tric & -0.015 & 0.365 & & 0.681 & 0.250 & 0.651 & 0.585 & 0.458 & 0.302 & 0.578 \\
Subs & 0.096 & 0.534 & 0.688 & & 0.448 & 0.703 & 0.720 & 0.449 & 0.382 & 0.602 \\
FFM & 0.813 & 0.858 & 0.109 & 0.261 & & 0.308 & 0.464 & -0.199 & 0.645 & -0.016 \\
FM & 0.242 & 0.582 & 0.537 & 0.624 & 0.082 & & 0.709 & 0.853 & 0.045 & 0.924 \\
BMI & -0.192 & 0.448 & 0.598 & 0.696 & 0.188 & 0.572 & & 0.472 & 0.677 & 0.692 \\
\%FM & -0.135 & 0.154 & 0.438 & 0.428 & -0.370 & 0.885 & 0.442 & -0.313 & 0.958 \\
FFMI & -0.069 & 0.305 & 0.199 & 0.289 & 0.520 & -0.231 & 0.591 & -0.458 & -0.053 \\
FMI & -0.166 & 0.261 & 0.537 & 0.571 & -0.252 & 0.907 & 0.662 & 0.959 & -0.211 \\
\hline
\end{tabular}

upper, boys; lower, girls; $\mathrm{r}>0.138, \mathrm{p}<0.05 ; \mathrm{r}>0.181, \mathrm{p}<0.01$

ST, stature; BM, body mass; Tric, triceps; Subs, subscapular; FFM, fat-free mass; FM, fat mass; BMI, body mass index; \%FM, percent fat mass; FFMI, fatfree mass index; FMI, fat mass index

for FMI were the same as for BMI using the same sample. Because the \%FM is now used widely to measure obesity, and both BMI and FMI were compared with the \%FM.

The statistical analysis program StatView, version J-4.5 was used for statistical analysis. The results are expressed as mean \pm standard deviation. Data for boys and girls were analyzed separately. Gender and group differences in physical characteristics and body composition were examined by unpaired $t$-test. The interrelations among stature, body mass, triceps, sub-scapular, FFM, FM, BMI, \%FM, FFMI, and FMI were examined using Pearson correlation. The relationships between BMI and stature or \%FM, and between FMI and stature or $\% \mathrm{FM}$, were also assessed using regression analysis. Differences with $P$ values less than 0.05 were considered significant.

\section{Results}

The inter-correlations of stature, body mass, skin-folds, FFM, FM, BMI, \%FM, FFMI, and FMI are presented in Table 2. The $\% \mathrm{FM}$ was not strongly correlated with stature $(r=-0.090$ in boys and -0.135 in girls) or body mass $(r=0.212$ in boys and 0.154 in girls). The $\% \mathrm{FM}$ was more strongly correlated with FMI $(r=0.958$ in boys and 0.959 in girls; $p<0.001)$ and was moderately correlated with BMI $(r=0.472$ in boys and 0.442 in girls; $p<0.001)$. Plots of stature and \%FM against BMI and FMI are given in Fig. 1 and Fig. 2. There were no significant correlations between BMI and stature or between FMI and stature, but there were correlations between BMI and \%FM or between FMI and \%FM in both boys and girls. As shown in Fig. 2, FMI was significantly correlated with $\% \mathrm{FM}$ in boys and girls with similar regression slopes. The entire study sample was classified into 3 groups according to indicators of obesity (i.e., \%FM, BMI, and FMI). Table 3 gives the descriptive statistics on the obese boys and girls according to each criterion. The unpaired $t$-test was used to evaluate the group differences for \%FM vs BMI and FMI. When the 90 percentile of BMI was used to define obesity,

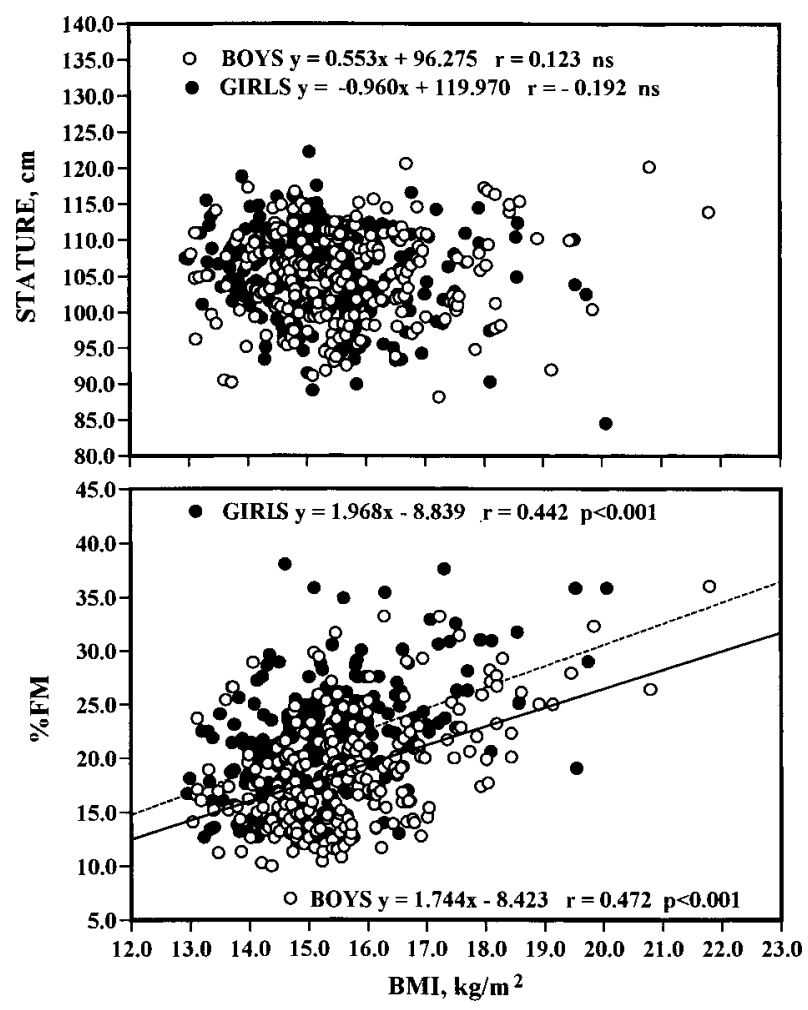

Fig. 1 Stature and percent fat mass $(\% \mathrm{FM})$ as a function of body mass index (BMI).

BMI for both boys and girls was significantly higher compared with using the $\% \mathrm{FM}$ criterion. When the 90th percentile of FMI was used to define obesity, FMI in girls was significantly higher compared with using the $\% \mathrm{FM}$ criterion, but the difference was not statistically significant in boys. Table 4 demonstrates the validity of using BMI or FMI to detect obesity. When the 90th percentile of BMI and FMI was used to define obesity, the prevalence was lower compared with using the $\% \mathrm{FM}$ criterion was used. Although the specificity of both indices was quite high in the populations, the definitions of obesity based on BMI and FMI were found to be somewhat 
insensitive to obesity as determined by BIA. The sensitivities of the FMI were $68.8 \%$ in boys and $42.9 \%$ in girls for $\% \mathrm{FM}$. BMI indicated obesity in less than half of the boys $(37.5 \%)$ and girls $(30.4 \%)$ who were defined as obese by the $\% \mathrm{FM}$ criterion. Although the false positive rates of BMI criterion as an obesity definition were $4.5 \%$ in boys and $3.6 \%$ in girls, the false negative rates for this definition were $62.5 \%$ and $69.6 \%$ in boys and girls, respectively. In contrast, the false negative rates of the FMI criterion were slightly lower, ranging from $31.2 \%$ in boys to $57.1 \%$ in girls for the obesity with $\% \mathrm{FM}$ as the criterion.

\section{Discussion}

The definition of childhood obesity remains unclear.
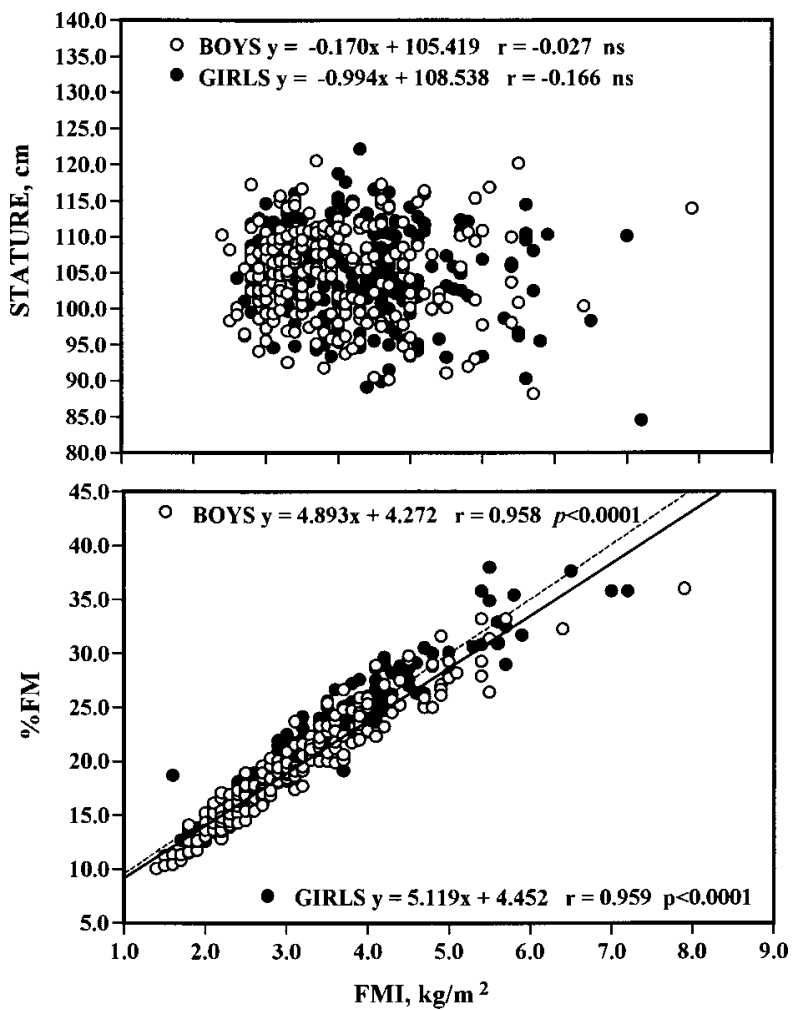

Fig. 2 Stature and percent fat mass $(\% \mathrm{FM})$ as a function of fat mass index (FMI).
Clinically, the presence of obesity in childhood is frequently assessed by BMI. The practical advantages of BMI as a measure of obesity are obvious. Both stature and body mass are easily and accurately measured with simple equipments. Although several studies have reported a high correlation between BMI and fatness (Sarria and Garcia-Llop, 1998; Chan et al., 1998; Pietrobelli and Faith, 1998), the statistical rationale for BMI is that it is an index of body mass that is minimally correlated with stature. Since stature is one component, the BMI may be stature-dependent over at least part of the age range. In particular, the assumption that the BMI is independent of stature is not true for children. However, Rolland-Cachera et al. (1982) have demonstrated that the coefficient of the correlation between BMI and stature are weak and sometimes negative from 1 to $6 \mathrm{yr}$. In the present study, there was a correlation between BMI and \%FM, but there was no significant correlation between BMI and stature in boys and girls aged $3-5 \mathrm{yr}$. Therefore, BMI in this study is a useful index with which to assess obesity and is a reasonable indicator of fatness.

The body mass comprises both FFM and FM, and both of these components can vary between each individual. Fomon's study (Fomon et al., 1982) describes the change in \%FM for average boys and girls from birth to age at $10 \mathrm{yr}$. However, $\% \mathrm{FM}$ is not independent of the quantity of FFM (Van Itallie et al., 1990; Hattori et al., 1997), so the normalization of FM in terms of body size provides only a crude method of comparing

Table 4 Validity of BMI and FMI as indicators of obesity compared to percent fat mass $(\% \mathrm{FM})$

\begin{tabular}{lccccc}
\hline & $\begin{array}{c}\text { Prevalence } \\
(\%)\end{array}$ & $\begin{array}{c}\text { Sensitivity } \\
(\%)\end{array}$ & $\begin{array}{c}\text { Specificity } \\
(\%)\end{array}$ & $\begin{array}{c}\text { False } \\
\text { negatives }\end{array}$ & $\begin{array}{c}\text { False } \\
\text { positives }\end{array}$ \\
\hline BMI & & & & & \\
Boys & 9.0 & 37.5 & 95.5 & 62.5 & 4.5 \\
Girls & 6.7 & 30.4 & 96.4 & 69.6 & 3.6 \\
$F M I$ & & & & & \\
Boys & 9.9 & 68.8 & 99.5 & 31.2 & 0.5 \\
Girls & 9.5 & 42.9 & 100 & 57.1 & 0 \\
\hline
\end{tabular}

Prevalence of obesity based on $\% \mathrm{FM}$, boys $=13.7$; girls $=22.1$.

Sensitivity, proportion diagnosed obese by the BMI if obese by \%FM; Specificity, proportion diagnosed lean by the BMI if lean by \%FM; False negative, 100\%-sensitivity; False positive, 100\%—-specificity.

Table 3 Means and standard deviations of stature, body mass, \%FM, BMI, and FMI in obese children by each obesity indicator

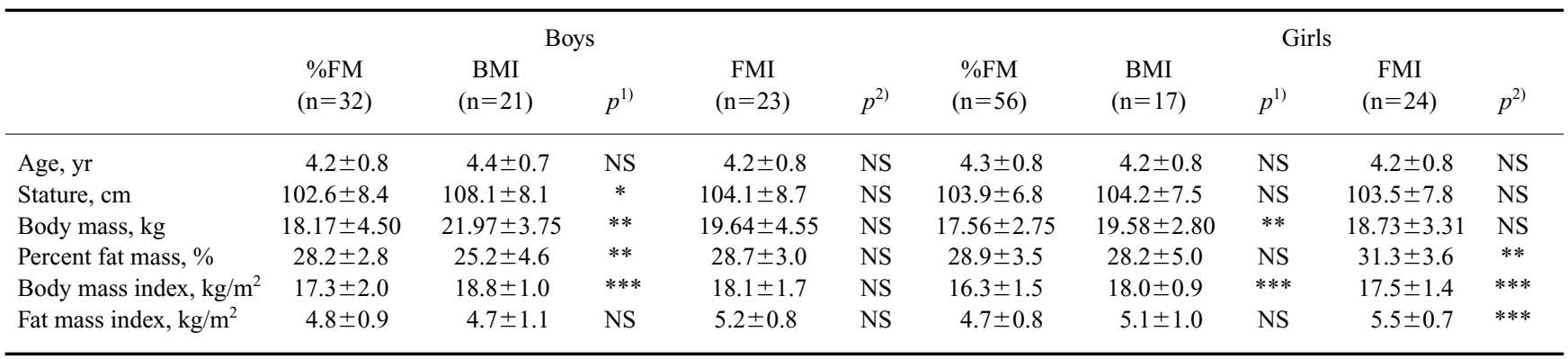

Group difference, ${ }^{1)} \% \mathrm{FM}$ vs BMI; ${ }^{2)} \% \mathrm{FM}$ vs FMI; * $p<0.05 ; * * p<0.01 ; * * * p<0.001$ 
fatness between each individual. BMI represents an index of body mass that has been normalized for stature. Once the body mass has been normalized in this way, it can be divided into FFM/stature ${ }^{2}$ and FM/stature ${ }^{2}$. These two indices have been termed FFMI and FMI, respectively (Van Itallie et al., 1990). However, this normalization using stature $^{2}$ is based on the relationship between the body mass and stature, and not on the separate relationships between FFM and stature and between FM and stature. Such separate evaluations would be required in order to generate indices of FFM and FM that each has a minimal correlation with stature. Because FM is more variable between each individual than FFM, the two components of body mass cannot be assumed to be normalized for stature in exactly the same way. In the present study, FMI was significantly correlated with $\% \mathrm{FM}$ in boys and girls with similar regression slopes, but there was no significant correlation between FMI and stature in boys or girls aged 3-5 yr. Therefore, FMI in this study is also useful index with which to assess obesity and is a reasonable indicator of fatness.

The purpose of the present study is to evaluate the sensitivity and specificity of BMI and FMI as indicators of obesity in a large sample of children aged 3-5 yr. In general, anthropometrical indicators of obesity are characterized by low sensitivities and high specificities (Himes and Bouchard, 1989). In this study, the specificities of BMI relative to $\% \mathrm{FM}$ were high, but the sensitivities were low, ranging from $30.4 \%$ to $37.5 \%$ for obesity with $\% \mathrm{FM}$ as the criterion. In contrast, the sensitivities of the FMI relating to $\% \mathrm{FM}$ were somewhat high, ranging from $42.9 \%$ in girls to $68.8 \%$ in boys. Accordingly, the majority of truly obese children are not correctly identified using the BMI, but almost all children who are not obese are correctly identified. This was also true for FMI. In the samples (aged from 3 to $5 \mathrm{yr}$ ) from the present study, the BMI and FMI were poor predictors of obesity compared with \%FM. Perhaps the cutoff values for obesity based on $\% \mathrm{FM}$ (i.e., $\geqq 20 \% \mathrm{FM}$ in boys; $\geqq 25 \% \mathrm{FM}$ in girls) were low or, conversely, the cutoff values for obesity based on the BMI (i.e., $\geqq 90$ th percentile in boys and girls) were too high such that the criteria did not appropriately reflect a safe amount of total body fatness. These \%FM were lower than those used by Lohman (i.e., $\geqq 25 \% \mathrm{FM}$ in boys; $\geqq 30 \% \mathrm{FM}$ in girls) (Lohman, 1992). In the present study, the 90th percentile for BMI distribution corresponded to the cutoff levels of $\% \mathrm{FM}$ of 25.2 in boys and 28.2 in girls. This is particularly true in girls: at the 90th percentile for BMI the corresponding $\% \mathrm{FM}$ is $<30 \%$ FM. Therefore, the prevalence of obesity based on BMI was lower than that based on \%FM.

Surprisingly, FMI has not yet found a wide application, probably because appropriate reference standards have yet to be defined. One advantage of FMI, as compared to the BMI concept, is that it amplifies the relative effect of growth on FM. The expression of a change in FM in absolute value fails to allow an appropriate comparison among subjects of different body size. Considering that BMI is the sum of FFMI+FMI, an increase in BMI could be accounted for by a rise in one component or the other or in both components. Note that, for a given BMI, if FMI increases then FFMI should decrease, since, at a constant BMI, there is an inverse mathematical relationship between the two. Therefore, the advantage of the combined use of these indices is that one can judge whether the excess of body mass is selectively due to a change in FM vs FFM or both combined. However, up to now, FMI cutoff points for obesity have not been clearly defined, at least not in a large group of Japanese children. In the present study, the 90th percentile for FMI distribution corresponded to the FMI cutoff points of 5.2 in boys and 5.5 in girls.

In summary, while the sensitivity of FMI in the samples from the present study was somewhat high, the definition of childhood obesity based on BMI had a low sensitivity. However, in screening for obese children, the specificity may be more important than the sensitivity. Maximizing specificity minimizes the proportion of children who will be incorrectly considered obesity by the screen. In the present study, the 90th percentile cutoff points for BMI and FMI had very high specificities. However, the partitioning of BMI into FFMI and FMI is obviously not possible without the associated measurements of body composition. Future investigations including body composition measurements will help to elucidate the relationship between the magnitude of FMI and potential risk factors. The present study assessed the validity of BMI and FMI as indicators of obesity in children, but it definitely warrants complementary investigations in a large group of Japanese children. This article is a preliminary attempt to promote future research in the area of childhood obesity. Furthermore, the concept of FMI could also be developed for obese children although less information on body composition is available for young children.

\section{References}

Chan YL, Leung SSF, Lam WWM, Peng XH, Metreweli C (1998) Body fat estimation in children by magnetic resonance imaging, bioelectrical impedance, skinfold and body mass index: A pilot study. J Paediatr Child Health 34: $22-28$

Dietz WH, Robinson TN (1988) Use of the body mass index (BMI) as a measure of overweight in children and adolescents. J Pediatr 132: 191-193

Fomon SJ, Haschke F, Ziegler EE, Nelson SE (1982) Body composition of reference children from birth to age 10 years. Am J Clin Nutr 35: 1169-1175

Forbes GB, Reina JC (1970) Adult lean body mass declines with age: Some longitudinal observations. Metabolism 19: 653-663

Garn SM, Leonard WR, Hawthorne VM (1986) Three limitations of the body mass index. Am J Clin Nutr 44: 996-997

Goran MI, Kaskoun MC, Carpenter WH, Poehlman ET, Ravussin E, Fontviekke A-M (1993) Estimating body composition of young children by using bioelectrical 
resistance. J Appl Physiol 75: 1776-1780

Griffiths M, Payne PR, Stunkard AJ, Rivers JPW, Cox M (1990) Metabolic rate and physical development in children at risk of obesity. Lancet 336: 76-78

Guillaume M (1999) Defining obesity in childhood: Current practice. Am J Cln Nutr 70 (Suppl): 126s-130s

Hattori K, Tatsmi N, Tanaka S (1997) Assessment of body composition by using a new chart method. Am J Hum Biol 9: 573-578

Himes JH, Bouchard C (1989) Validity of anthropometry in classifying youths as obese. Int J Obes 13: 183-193

Himes JH, Dietz WH (1994) Guidelines for overweight in adolescent preventive services: Recommendations from an expert committee. Am J Clin Nutr 59: 307-316

Houtkooper LB, Lohman TG, Going SB, Howell WH (1996) Why bioelectrical impedance analysis should be used for estimating adiposity. Am J Clin Nutr 64: 436S-448S

Lohman TG (1992) Advances in Body Composition Assessment. Human Kinetics, Champaign

Lukaski HC (1986) Validation of tetrapolar bioelectrical impedance measurements to assess human body composition. J Appl Physiol 60: 1327-1332

Marshall JD, Hazlett CB, Spady DW, Conger PR, Quinney HA (1991) Validity of convenient indicators of obesity. Hum Biol 63: 137-153

Owen OE (1988) In: Kinney JM, Jeejeebhoy KN, Hill GL, Owen OE, eds. Nutritional and Metabolism in Patient Care. Philadelphia; WB Saunders, 1081-1107

Pietrobelli A, Faith MS (1998) Body mass index as a measure of adiposity among children and adolescents: A validation study. J Pediatr 132: 204-210
Reilly JJ, Dorosty AR (1999) Epidemic of obesity in UK children. Lancet 354: 1874-1875

Rolland-Cachera MF, Sempe M, Guilloud-Bataille M, Patois E, Pequignot-Guggenbuhl F, Fautrad V (1982) Adiposity indices in children. Am J Clin Nutr 36: 178-184

Sarri A, Garcia-Llop LA (1998) Skinfold thickness measurements are better predictors of body fat percentage than body mass index in male Spanish children and adolescents. Eur J Clin Nutr 52: 573-576

Troiano RP, Flegal KM, Kuczmarski RJ, Campbell SM, Johnson CL (1995) Overweight prevalence and trends for children and adolescents: The National Health and Nutrition Examination Surveys, 1963 to 1991. Arch Pediatr Adolesc Med 149: 1085-1091

Van Itallie TB, Yang M-U, Heymsfield SB, Funk RC, Boileau R (1990) Height-normalized indices of the body's fat-free mass and fat mass: Potentially useful indicators of nutritional status. Am J Clin Nutr 52: 953-959

Wells JCK (2000) Hattori chart analysis of body mass index in infants and children. Int $\mathrm{J}$ Obes Relat Metab Disord 24: 325-329

Received: June 2, 2003

Accepted: December 18, 2003

Correspondence to: Shuichi Komiya, Graduate School of Human-Environment, Kyushu University, 6-1 Kasugakouen, Kasuga 816-8580, Japan

Phone: +81-92-5837848

Fax: +81-92-5837848

e-mail: komiya@ihs.kyushu-u.ac.jp 